\title{
The Transmuted Inverted Nadarajah-Haghighi Distribution With an Application to Lifetime Data
}

\author{
Aliyeh Toumaj $^{1}$, S.M.T.K. MirMostafaee ${ }^{2 *}$, \\ G.G. Hamedani ${ }^{3}$ \\ ${ }^{*}$ Corresponding author
}

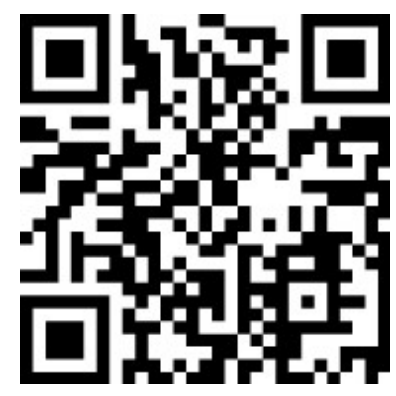

1. Department of Statistics, University of Mazandaran, Babolsar, Iran, aliye_tomaj@yahoo.com

2. Department of Statistics, University of Mazandaran, Babolsar, Iran, m.mirmostafaee@umz.ac.ir

3. Marquette University, Milwaukee, WI 53201-1881, USA, g.hamedani@mu.edu

\begin{abstract}
In this paper, we propose a new lifetime distribution. We discuss several mathematical properties of the new distribution. Certain characterizations of the new distribution are provided. We study the maximum likelihood estimation and asymptotic interval estimation of the unknown parameters. A simulation study, as well as an application of the new distribution to failure data, are also presented. We end the paper with a number of remarks.
\end{abstract}

Key Words: Maximum Likelihood Estimation; Goodness-of-Fit Criteria; Quadratic Rank Transmutation Map; Reversed Hazard Rate Function; Simulation.

Mathematical Subject Classification: 60E05, 62E15, 62F10.

\section{Introduction}

Lifetime distributions play important roles in modeling and analysis of many life phenomena. Consequently, many researchers have tried to introduce new competitive lifetime distributions in order to model real data that arise in many areas such as medicine, engineering and economics where the old distributions cannot be employed. Generalizing a lifetime distribution often consists of adding one or more parameters to the baseline distribution. The new parameters could make the generalized distribution more flexible in the sense that it can model the data sets more suitably. There exist many methods of generalizing distributions, see for example Lee et al. (2013) for a discussion regarding a variety of methods of generating statistical distributions developed in recent decades. Among the generalizing methods, an approach, called the quadratic rank transmutation map, has been proposed by Shaw and Buckley (2007) and Shaw and Buckley (2009). Let $g(x)$ and $G(x)$ be the probability density function (pdf) and cumulative distribution function (cdf) of the baseline distribution, respectively, of a random variable $X$, then the cdf and pdf of the transmuted family of distributions are given by

$$
F(x)=(1+\lambda) G(x)-\lambda G(x)^{2}, \quad|\lambda| \leq 1, \quad x \in \mathbb{R},
$$

and

$$
f(x)=(1+\lambda) g(x)-2 \lambda g(x) G(x), \quad x \in \mathbb{R}
$$

respectively. 
Many authors use the transmutation procedure to achieve new generalized distributions, see for example the transmuted generalized inverse Weibull distribution (Khan and King, 2014, and Merovci et al., 2013), the transmuted exponentiated exponential distribution (Merovci, 2013a), the transmuted Lindley distribution (Merovci, 2013b), the transmuted Dagum distribution (Elbatal and Aryal, 2015), the transmuted Burr type III distribution (Abdul-Moniem, 2015) and the transmuted Birnbaum-Saunders distribution (Bourguignon et al., 2017).

Nadarajah and Haghighi (2011) introduced an extension of the exponential distribution, called the Nadarajah-Haghighi distribution, with the following pdf

$$
f_{N H}(x)=\alpha \beta(1+\beta x)^{\alpha-1} \mathrm{e}^{1-(1+\beta x)^{\alpha}}, \quad x>0, \alpha, \beta>0 .
$$

Here, we emphasize that the Nadarajah-Haghighi distribution is a special case of the distribution introduced by Dimitrakopoulou et al. (2007).

Tahir et al. (2018) used the transformation $Y=1 / X$, where $X$ has a Nadarajah-Haghighi distribution, to propose a new inverted model called the inverted Nadarajah-Haghighi (INH) distribution. The pdf of the INH distribution is given by

$$
g(y)=\frac{\alpha \beta}{y^{2}}\left(1+\frac{\beta}{y}\right)^{\alpha-1} \exp \left[1-\left(1+\frac{\beta}{y}\right)^{\alpha}\right], \quad y>0, \quad \alpha, \beta>0 .
$$

The corresponding cdf of the INH is

$$
G(y)=\exp \left[1-\left(1+\frac{\beta}{y}\right)^{\alpha}\right], \quad y \geq 0
$$

In this paper, we use the transmutation map to generalize the INH distribution. To this end, we take $g(x)$ and $G(x)$ in (2) to be the pdf and cdf of the INH distribution, respectively, to obtain the pdf of the transmuted inverted NadarajahHaghighi (TINH) distribution as follows

$$
f(x)=\frac{\alpha \beta}{x^{2}}\left(1+\frac{\beta}{x}\right)^{\alpha-1} \exp \left[1-\left(1+\frac{\beta}{x}\right)^{\alpha}\right]\left\{\begin{array}{r}
\left.1+\lambda-2 \lambda \exp \left[1-\left(1+\frac{\beta}{x}\right)^{\alpha}\right]\right\}, \\
\alpha>0, \quad \beta>0, \quad|\lambda| \leq 1, \quad x>0 .
\end{array}\right.
$$

We write $X \sim \operatorname{TINH}(\alpha, \beta, \lambda)$ if the pdf of $X$ is (3). The corresponding cdf of the TINH distribution is given by

$$
F(x)=\exp \left[1-\left(1+\frac{\beta}{x}\right)^{\alpha}\right]\left\{1+\lambda-\lambda \exp \left[1-\left(1+\frac{\beta}{x}\right)^{\alpha}\right]\right\}, \quad x \geq 0 .
$$

The special cases of the TINH distribution are listed below:

- For $\lambda=0$, we obtain the INH distribution (Tahir et al., 2018).

- For $\alpha=1$, we obtain the transmuted inverted (inverse) exponential distribution, discussed by Oguntunde and Adejumo (2015).

- For $\lambda=0$ and $\alpha=1$, we obtain the inverted (inverse) exponential distribution.

In addition, the hazard rate function (hrf) of the new distribution turns out to be

$$
h(x)=\frac{\frac{\alpha \beta}{x^{2}}\left(1+\frac{\beta}{x}\right)^{\alpha-1} \exp \left[1-\left(1+\frac{\beta}{x}\right)^{\alpha}\right]\left\{1+\lambda-2 \lambda \exp \left[1-\left(1+\frac{\beta}{x}\right)^{\alpha}\right]\right\}}{1-\exp \left[1-\left(1+\frac{\beta}{x}\right)^{\alpha}\right]\left\{1+\lambda-\lambda \exp \left[1-\left(1+\frac{\beta}{x}\right)^{\alpha}\right]\right\}}, \quad x>0 .
$$

Figures 1 and 2 show the shapes of (3) and (5) for selected values of the parameters, respectively. From Figure 1, we see that the pdf of the new model is unimodal. Figure 2 reveals that the hrf shapes are upside-down bathtub (increasing-decreasing). Therefore the new distribution shows a somehow flexibility and it may be used to model the lifetime data sets that cannot be modeled by non-inverted distributions satisfactorily. Here, we emphasize that hrf might not be absolutely decreasing. Tahir et al. (2018) claimed that the pdf and hrf of the INH distribution can be 
decreasing and they plotted pdf and hrf when the scale and shape parameters of the INH distribution set equal to one and 0.4 , respectively, to show their claim. However, we plotted the pdf and hrf of the INH distribution (recall that INH is a special case of TINH when $\lambda=0$ ) and observed that they both are increasing-decreasing, see Figure 3.

$\alpha=0.5 ; \beta=1$

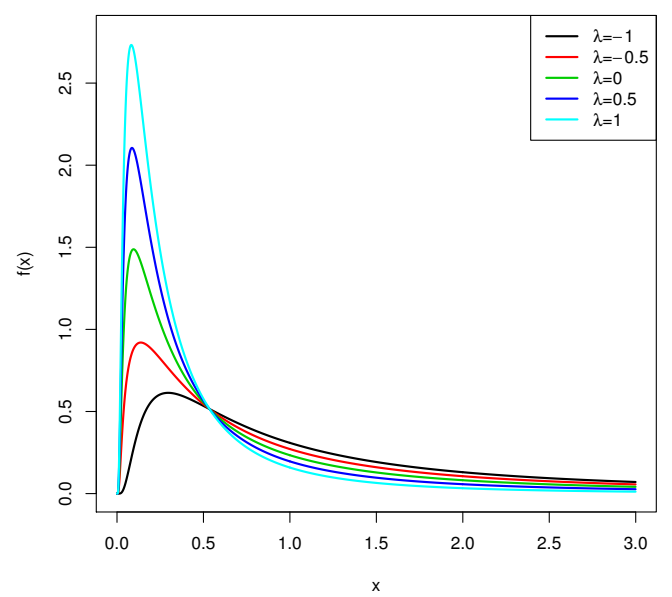

$\alpha=3 ; \beta=1$

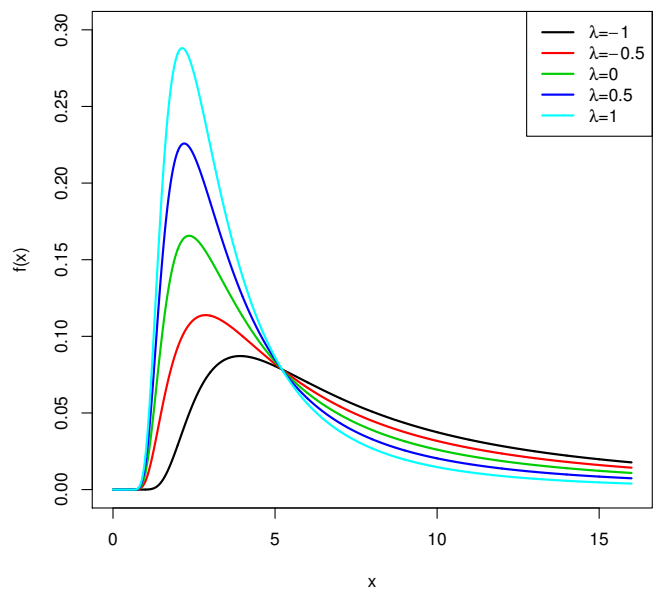

Figure 1: Plots of the pdfs of the TINH distribution for selected parameter values.
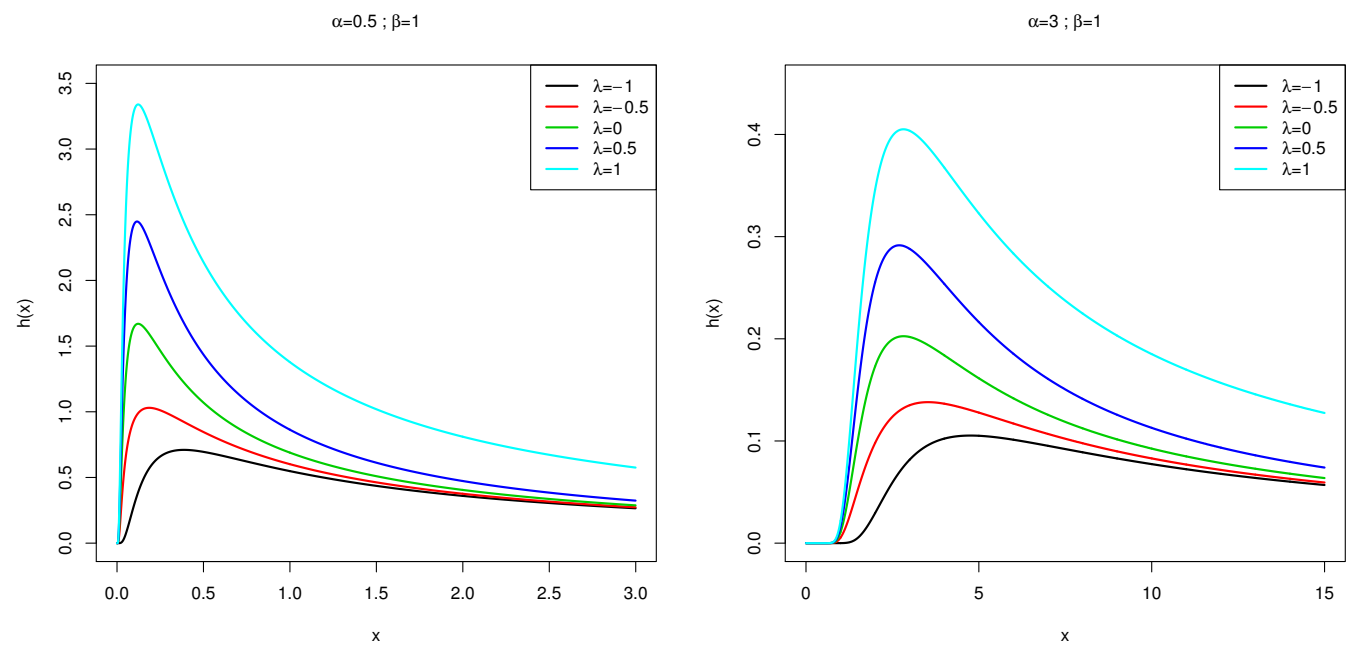

Figure 2: Plots of the hrfs of the TINH distribution for selected parameter values.

The rest of the article is organized as follows: Several mathematical properties of the new model are presented in Section 2. Certain characterizations of the new distribution are provided in Section 3. Section 4 is devoted to maximum likelihood estimation of the parameters, a discussion regarding the asymptotic behavior of the maximum likelihood estimators and a simulation study. An application of the new model is provided in Section 5. Finally, a number of remarks are given in Section 6.

\section{Mathematical Properties}

In this section, we focus on some mathematical properties of the new distribution, such as the quantile function, skewness, kurtosis, moments, reversed hazard rate function, the reliability parameter and order statistics. 

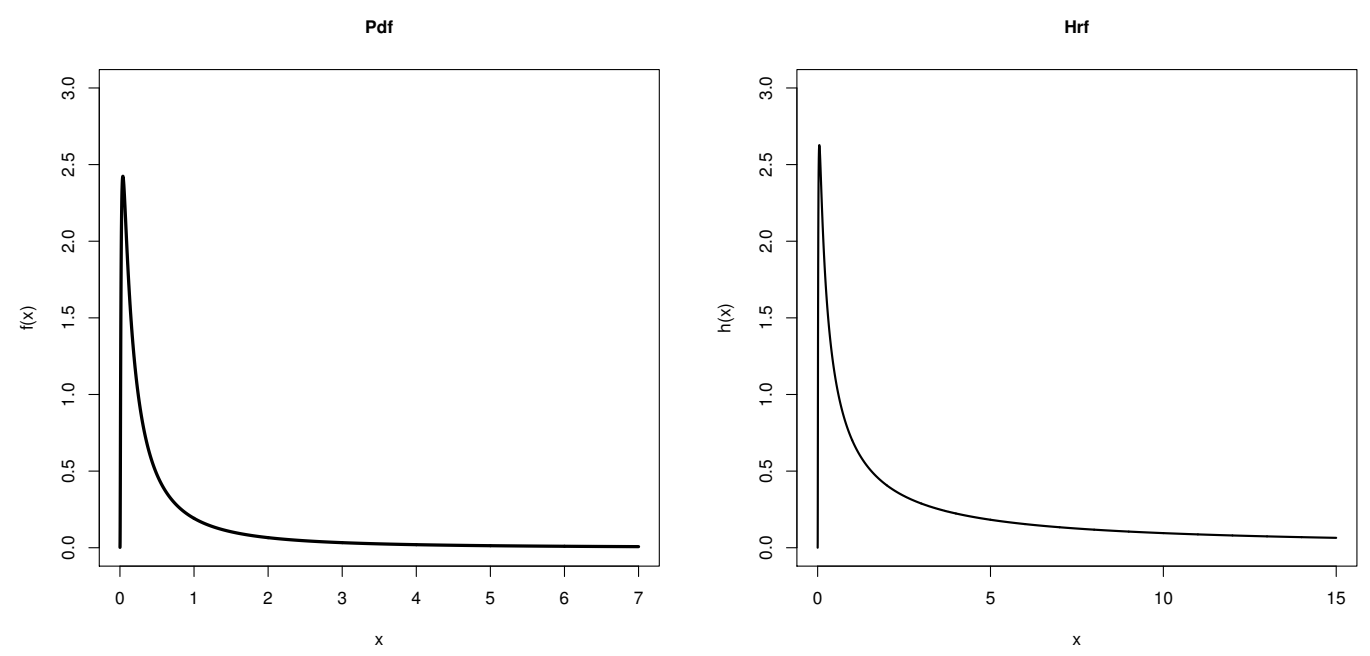

Figure 3: Plots of the pdf (left) and hrf (right) of the TINH distribution when $\beta=1, \alpha=0.4$ and $\lambda=0$.

\subsection{Quantile function}

The quantile function is one of the important characteristics of a distribution. One application of the quantile function arises when one wants to generate samples from the selected distribution. For the TINH distribution, the quantile function $x=Q(p)$, can be derived via inverting the cdf (4)

$$
Q(p)= \begin{cases}\beta\left[\left(1-\ln \left[\frac{(1+\lambda)-\sqrt{(1+\lambda)^{2}-4 \lambda p}}{2 \lambda}\right]\right)^{\frac{1}{\alpha}}-1\right]^{-1}, & \text { if } \quad \lambda \neq 0, \\ \beta\left[(1-\ln p)^{\frac{1}{\alpha}}-1\right]^{-1}, & \text { if } \quad \lambda=0 .\end{cases}
$$

The median of $X \sim \operatorname{TINH}(\alpha, \beta, \lambda)$ is simply obtained by setting $p=0.5$ in (6). In order to generate a random sample of size $n$ from $\operatorname{TINH}(\alpha, \beta, \lambda)$, first generate a random sample, say $U_{1}, \cdots, U_{n}$ from the standard uniform distribution, then the required random sample of size $n, X_{1}, \cdots, X_{n}$, can be derived by using the relation $X_{i}=Q\left(U_{i}\right)$ for $i=1, \cdots, n$.

We can also obtain the measures of skewness and kurtosis based on the quantile function. The Bowley skewness (also known as the Galton skewness) is formulated based on quartiles. Let $Q_{i}$ be the $i$-th quartile of a distribution $(i=1,2,3)$, then the Bowley skewness is given by

$$
\mathcal{B}=\frac{Q_{3}+Q_{1}-2 Q_{2}}{Q_{3}-Q_{1}}=\frac{Q\left(\frac{3}{4}\right)+Q\left(\frac{1}{4}\right)-2 Q\left(\frac{2}{4}\right)}{Q\left(\frac{3}{4}\right)-Q\left(\frac{1}{4}\right)} .
$$

The plots of the Bowley skewness with respect to (w.r.t.) $\alpha$ for some values of $\lambda$ and w.r.t. $\lambda$ for some values of $\alpha$, are displayed in Figure 4. Note that the value of $\beta$ does not affect $\mathcal{B}$ as $\beta$ is a scale parameter. Figure 4 reveals that the Bowley skewness is decreasing w.r.t. $\alpha$ but it is increasing-decreasing or decreasing-increasing-decreasing w.r.t. $\lambda$ (when the other two parameters are kept fixed). The positive values of $\mathcal{B}$ confirm that the TINH distribution is right-skewed for the selected parameter values.

Next, we consider the Moors kurtosis (see Moors, 1988), which is formulated based on the octiles. Let $O_{i}$ be the $i$-th octile of a distribution $(i=1, \cdots, 7)$, then the Moors kurtosis is

$$
\mathcal{M}=\frac{\left(O_{3}-O_{1}\right)+\left(O_{7}-O_{5}\right)}{O_{6}-O_{2}}=\frac{Q\left(\frac{3}{8}\right)-Q\left(\frac{1}{8}\right)+Q\left(\frac{7}{8}\right)-Q\left(\frac{5}{8}\right)}{Q\left(\frac{6}{8}\right)-Q\left(\frac{2}{8}\right)} .
$$

Figure 5 contains the plots of the Moors kurtosis w.r.t. $\alpha$ for some values of $\lambda$ and w.r.t. $\lambda$ for some values of $\alpha$. We note that the value of $\beta$ does not affect $\mathcal{M}$ either. From Figure 5, we see that the Moors kurtosis is decreasing w.r.t. $\alpha$ and it is increasing-decreasing or decreasing-increasing-decreasing w.r.t. $\lambda$ (when the other two parameters are kept 

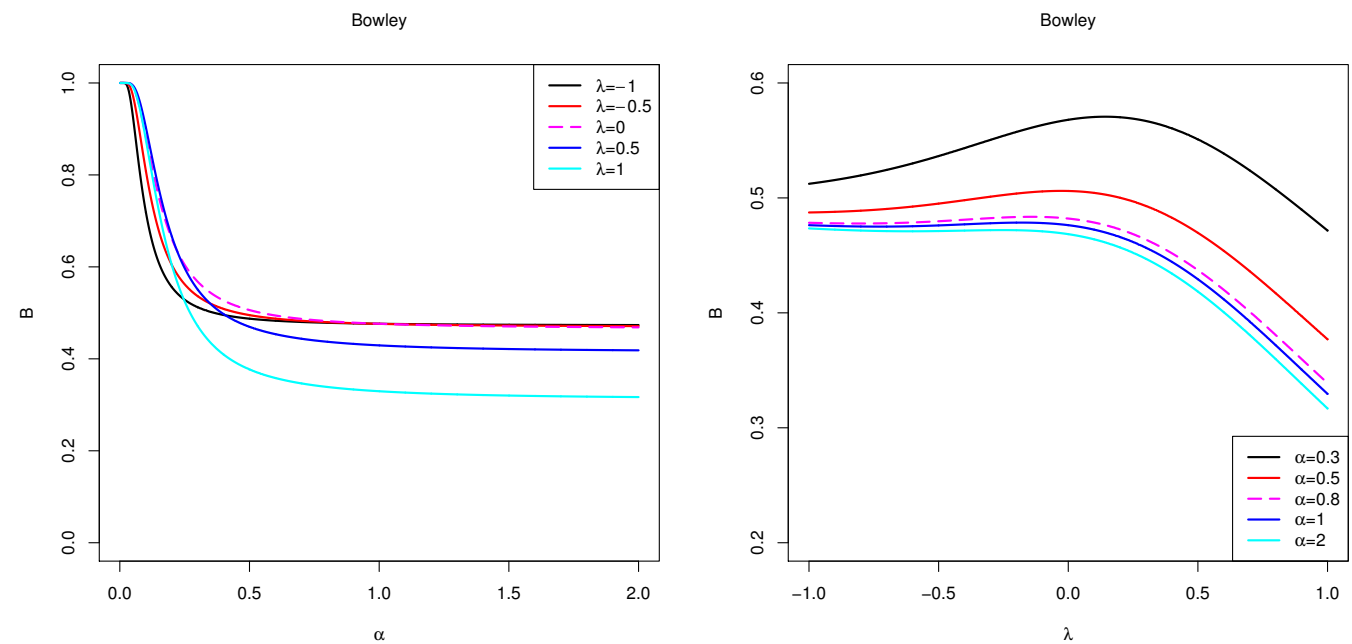

Figure 4: Plots of the Bowley skewness $(\mathcal{B})$ for the TINH distribution w.r.t $\alpha$ for some values of $\lambda$ (left) and w.r.t. $\lambda$ for some values of $\alpha$ (right).
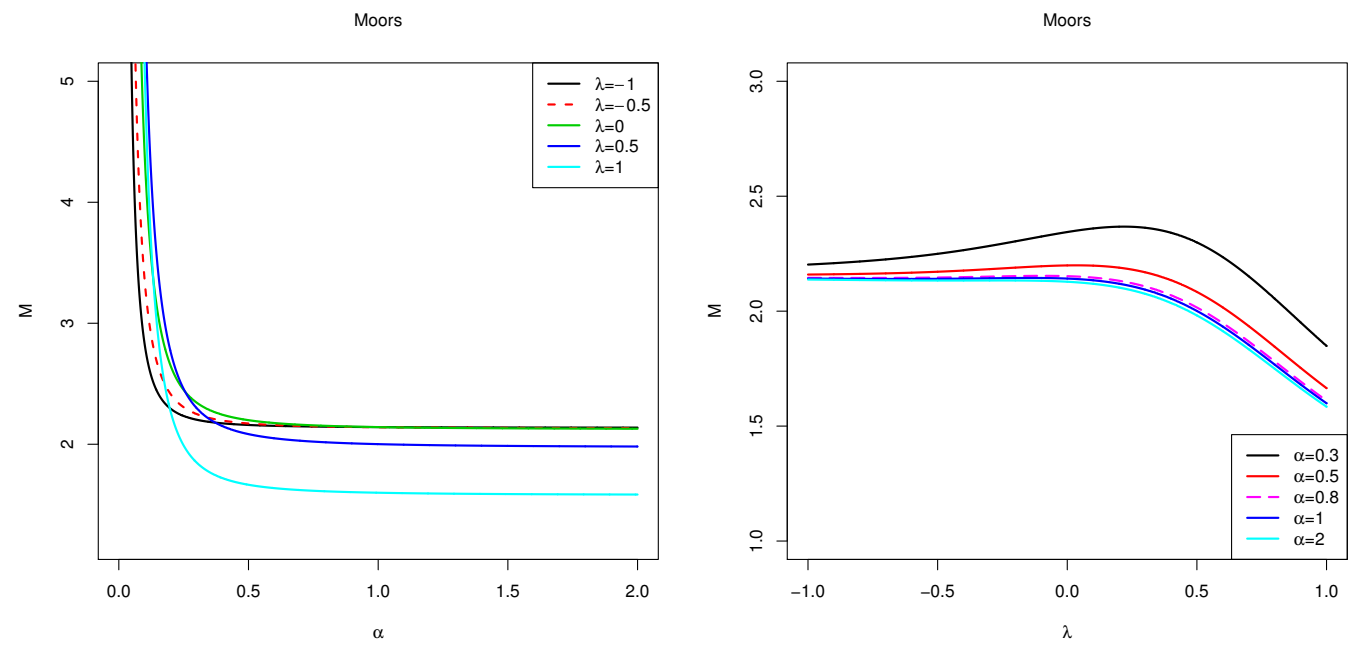

Figure 5: Plots of the Moors kurtosis $(\mathcal{M})$ for the TINH distribution w.r.t $\alpha$ for some values of $\lambda$ (left) and w.r.t. $\lambda$ for some values of $\alpha$ (right).

fixed).

Both measures of Bowley skewness and Moors kurtosis are not very sensitive to outliers. Another advantage of these measures is that they can be obtained regardless of the existence of the moments. Moreover, they are simply formulated and therefore they can easily be derived especially when the quantile function possesses an explicit form.

\subsection{Moments}

The $r$-th moment of the TINH distribution can be expressed as follows

$$
E\left(X^{r}\right)=\int_{0}^{\infty} x^{r-2} \alpha \beta\left(1+\frac{\beta}{x}\right)^{\alpha-1} \exp \left[1-\left(1+\frac{\beta}{x}\right)^{\alpha}\right]\left\{1+\lambda-2 \lambda \exp \left[1-\left(1+\frac{\beta}{x}\right)^{\alpha}\right]\right\} \mathrm{d} x
$$

Oguntunde and Adejumo (2015) emphasized that the first moment, second moment and the other higher-order moments do not exist for the transmuted inverse exponential distribution (a special case of the TINH distribution when $\alpha=1$ ). Therefore we can state that the ordinary moments (i.e. $E\left(X^{r}\right)$ for $r=1,2,3, \cdots$ ) of the TINH distribution do not exist for all combinations of the parameters. Actually, the existence of the $r$-th moment $(r=1,2,3, \cdots)$ depends 
on the parameter values. Tahir et al. (2018) reported a general expression for the $r$-th ordinary moment of the INH distribution and then stated that the measures of skewness and kurtosis can be obtained using these ordinary moments without noting the fact that the ordinary moments of the INH distribution might not exist for all the parameter combinations. Tahir et al. (2018) did not discuss the famous method of moment estimation of the parameters among the various estimation methods developed in their paper. Perhaps they could not apply the method of moment estimation because the ordinary moments of the INH distribution might not exist for all the parameter combinations.

We have implemented the computational softwares like $\mathrm{R}$ ( $\mathrm{R}$ Core Team, 2018) to check if the ordinary moments of the TINH distribution and its special cases exist. We set $\alpha=0.3,0.5,0.8,1,2,3,4, \beta=1, \lambda=-1,-0.5,0,0.5,1$ and $r=1,2$. Here, we recall that if $E\left(X^{2}\right)$ does not exist, then $E\left(X^{2+s}\right)$ for $s>0$ does not exist either. The software could not compute the moments except for the case $\lambda=1$ and $r=1$.

\subsection{Reversed hazard rate function}

The reversed hazard rate function (rhrf) of a continuous random variable $X$ is defined as

$$
r(t)=\lim _{\Delta t \rightarrow 0} \frac{P(X>t-\Delta t \mid X \leq t)}{\Delta t}=\frac{f(t)}{F(t)} .
$$

One property of the rhrf is that it cannot be increasing for non-negative distributions. In addition, the rhrf seems suitable for studying parallel systems, see Block et al. (1998). For the TINH distribution, we have

$$
r(t)=\frac{\frac{\alpha \beta}{t^{2}}\left(1+\frac{\beta}{t}\right)^{\alpha-1}\left\{1+\lambda-2 \lambda \exp \left[1-\left(1+\frac{\beta}{t}\right)^{\alpha}\right]\right\}}{1+\lambda-\lambda \exp \left[1-\left(1+\frac{\beta}{t}\right)^{\alpha}\right]}, \quad t>0 .
$$

We have plotted $r(t)$ for selected parameter values, see Figure 6. It can be seen that $r(t)$ is decreasing w.r.t. $t$ for all the considered cases in Figure 6.
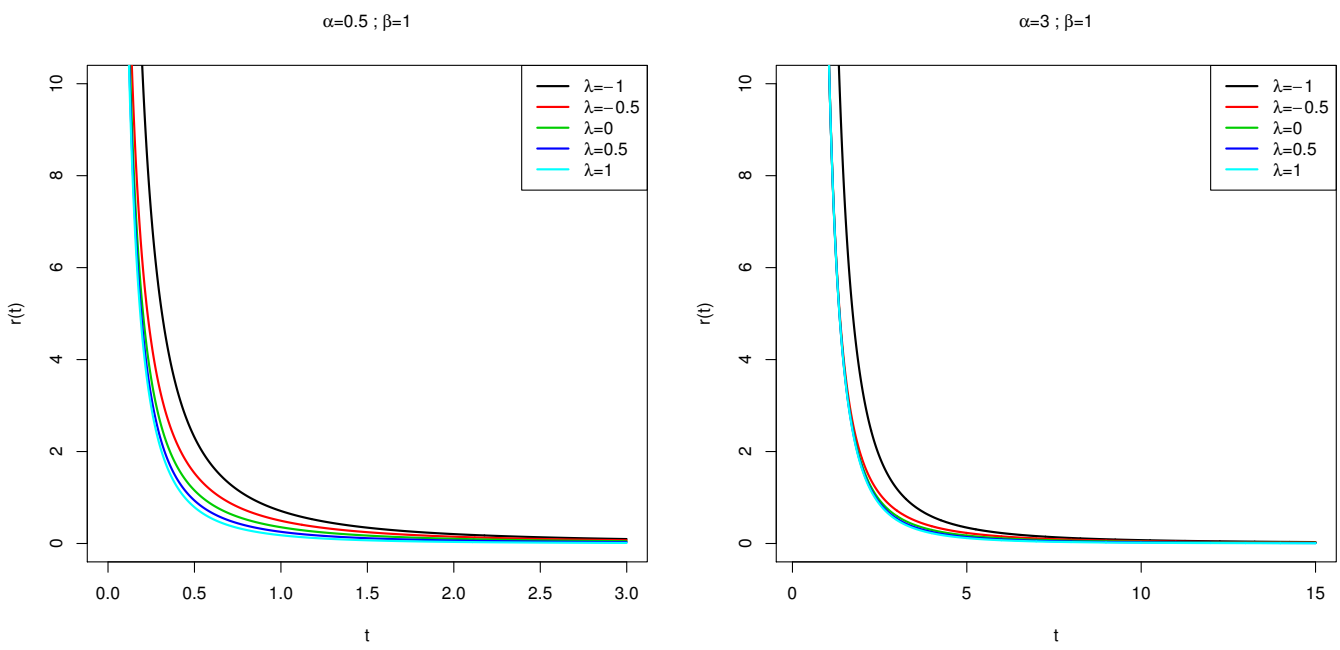

Figure 6: Plots of the rhrfs of the TINH distribution for some selected parameter values.

\subsection{Reliability parameter}

Let $X$ and $Y$ be two independent random variables. Then, the reliability parameter, also known as the stress-strength parameter, is simply defined as $P(X>Y)$. The stress-strength parameter may be interpreted as follows: Suppose $X$ denotes the strength of a component that is subjected to a stress $Y$. Then the component survives as long as $X$ remains greater than $Y$. The reliability parameter has other interpretations, as well. For example, it can be considered as a measure for the comparison of two populations. Discussion regarding the stress-strength parameter has absorbed many researchers and many statisticians studied the problems pertaining to the stress-strength parameter for various 
life distributions.

Now, suppose that $X \sim \operatorname{TINH}\left(\alpha, \beta, \lambda_{1}\right)$ and $Y \sim \operatorname{TINH}\left(\alpha, \beta, \lambda_{2}\right)$ are two independent random variables, then the stress-strength parameter is

$$
\begin{aligned}
R= & \int_{0}^{\infty} P(Y<X \mid X=x) f_{X}(x) \mathrm{d} x=\int_{0}^{\infty} f_{X}(x) F_{Y}(x) \mathrm{d} x \\
= & \int_{0}^{\infty} \frac{\alpha \beta}{x^{2}}\left(1+\frac{\beta}{x}\right)^{\alpha-1} \exp \left[1-\left(1+\frac{\beta}{x}\right)^{\alpha}\right]\left\{1+\lambda_{1}-2 \lambda_{1} \exp \left[1-\left(1+\frac{\beta}{x}\right)^{\alpha}\right]\right\} \\
& \times \exp \left[1-\left(1+\frac{\beta}{x}\right)^{\alpha}\right]\left\{1+\lambda_{2}-\lambda_{2} \exp \left[1-\left(1+\frac{\beta}{x}\right)^{\alpha}\right]\right\} \mathrm{d} x \\
= & \int_{0}^{1} u\left(1+\lambda_{1}-2 \lambda_{1} u\right)\left(1+\lambda_{2}-\lambda_{2} u\right) \mathrm{d} u \\
= & \frac{3-\lambda_{1}+\lambda_{2}}{6} .
\end{aligned}
$$

Setting $\lambda_{1}=\lambda_{2}$, we arrive at $R=0.5$ as expected.

\subsection{Order statistics}

Order statistics play a key role in many life experiments, especially when censoring schemes are applied. An application of order statistics arises when we deal with $k$-out-of- $n$ systems. Suppose that a system contains $n$ items and the lifetimes of the items, denoted by $X_{1}, \cdots, X_{n}$, are independent identically distributed with a common cdf $F$. Suppose further, the system works as long as at least $k$ items work. Let $X_{i: n}$ denote the $i$-th order statistic of the sample $X_{1}, \cdots, X_{n}$. Then the lifetime of the system coincides with $X_{n-k+1: n}$. Two special cases of $k$-out-of- $n$ systems are the parallel $(k=1)$ and series $(k=n)$ systems.

Here, we assume that the underlying cdf $F$, is the cdf of TINH distribution with parameters $\alpha, \beta$ and $\lambda$. In general, the cdf and pdf of the $i$-th order statistic are given by

$$
\begin{aligned}
F_{i: n}(x) & =\sum_{k=i}^{n}\left(\begin{array}{l}
n \\
k
\end{array}\right)[F(x)]^{k}\left[1-[F(x)]^{n-k}\right. \\
& =\int_{0}^{F(x)} \frac{n !}{(i-1) !(n-i) !} t^{i-1}(1-t)^{n-i} \mathrm{~d} t, \quad x \in \mathbb{R},
\end{aligned}
$$

and

$$
f_{i: n}(x)=\frac{n !}{(i-1) !(n-i) !}[F(x)]^{i-1}[1-F(x)]^{n-i} f(x), \quad x \in \mathbb{R},
$$

respectively.

Using the binomial expansion, we have

$$
[1-F(x)]^{n-i}=\sum_{k=0}^{n-i}(-1)^{k}\left(\begin{array}{c}
n-i \\
k
\end{array}\right)[F(x)]^{k} .
$$


Therefore, for the TINH distribution, we have

$$
\begin{aligned}
f_{i: n}(x) & =\sum_{k=0}^{n-i} \frac{(-1)^{k} n !}{k !(i-1) !(n-i-k) !}[F(x)]^{k+i-1} f(x) \\
& =\sum_{k=0}^{n-i} \frac{(-1)^{k} n !}{k !(i-1) !(n-i-k) !}\left[\exp \left[1-\left(1+\frac{\beta}{x}\right)^{\alpha}\right]\left\{1+\lambda-\lambda \exp \left[1-\left(1+\frac{\beta}{x}\right)^{\alpha}\right]\right\}\right]^{k+i-1} \\
& \times \frac{\alpha \beta}{x^{2}}\left(1+\frac{\beta}{x}\right)^{\alpha-1} \exp \left[1-\left(1+\frac{\beta}{x}\right)^{\alpha}\right]\left\{1+\lambda-2 \lambda \exp \left[1-\left(1+\frac{\beta}{x}\right)^{\alpha}\right]\right\} \\
& =\sum_{k=0}^{n-i} \sum_{j=0}^{k+i-1} \frac{(-1)^{k+j} n !\left(\begin{array}{c}
k+i-1 \\
j
\end{array}\right)(1+\lambda)^{k+i-1-j} \lambda^{j}}{k !(i-1) !(n-i-k) !} \exp \left[(k+i+j)\left(1-\left(1+\frac{\beta}{x}\right)^{\alpha}\right)\right] \\
& \times \frac{\alpha \beta}{x^{2}}\left(1+\frac{\beta}{x}\right)^{\alpha-1}\left\{1+\lambda-2 \lambda \exp \left[1-\left(1+\frac{\beta}{x}\right)^{\alpha}\right]\right\} .
\end{aligned}
$$

The pdf of the largest order statistic, corresponding to the lifetime of a parallel system, is then simplified as

$$
\begin{aligned}
f_{n: n}(x)= & n[F(x)]^{n-1} f(x) \\
= & \frac{n \alpha \beta}{x^{2}}\left(1+\frac{\beta}{x}\right)^{\alpha-1} \exp \left[n\left(1-\left(1+\frac{\beta}{x}\right)^{\alpha}\right)\right]\left\{1+\lambda-2 \lambda \exp \left[1-\left(1+\frac{\beta}{x}\right)^{\alpha}\right]\right\} \\
& \times\left\{1+\lambda-\lambda \exp \left[1-\left(1+\frac{\beta}{x}\right)^{\alpha}\right]\right\}^{n-1} .
\end{aligned}
$$

\section{Characterizations}

This section deals with various characterizations of the TINH distribution. These characterizations are based on: $(i)$ a simple relationship between two truncated moments and $(i i)$ the reversed hazard rate function.

\subsection{Characterizations based on two truncated moments}

In this subsection, we present characterizations of TINH distribution in terms of the ratio of two truncated moments. The first characterization result employs a theorem due to Glänzel (1987). Here, we restate the theorem.

Theorem 1: Let $(\Omega, \mathcal{F}, \mathbf{P})$ be a given probability space and let $H=[a, e]$ be an interval for some $a<e$ ( $a=$ $-\infty, e=\infty$ might as well be allowed). Let $X: \Omega \rightarrow H$ be a continuous random variable with the distribution function $F$ and let $q_{1}$ and $q_{2}$ be two real functions defined on $H$ such that

$$
E\left[q_{2}(X) \mid X \geq x\right]=E\left[q_{1}(X) \mid X \geq x\right] \xi(x), \quad x \in H,
$$

is defined with some real function $\xi$. Assume that $q_{1}, q_{2} \in C^{1}(H), \xi \in C^{2}(H)$ and $F$ is twice continuously differentiable and strictly monotone function on the set $H$. Finally, assume that the equation $\xi q_{1}=q_{2}$ has no real solution in the interior of $H$. Then $F$ is uniquely determined by the functions $q_{1}, q_{2}$ and $\xi$, particularly

$$
F(x)=\int_{a}^{x} C\left|\frac{\xi^{\prime}(u)}{\xi(u) q_{1}(u)-q_{2}(u)}\right| \exp (-s(u)) \mathrm{d} u,
$$

where the function $s$ is a solution of the differential equation $s^{\prime}=\frac{\xi^{\prime} q_{1}}{\xi q_{1}-q_{2}}$ and $C$ is the normalization constant, such that $\int_{H} \mathrm{~d} F=1$.

Note that the result holds also when the interval $H$ is not closed. As shown in Glänzel (1990), this characterization is stable in the sense of weak convergence.

Proposition 1: Let $X: \Omega \rightarrow(0, \infty)$ be a continuous random variable and let $q_{1}(x)=\left\{1+\lambda-2 \lambda \exp \left[1-\left(1+\frac{\beta}{x}\right)^{\alpha}\right]\right\}^{-1}$ 
and $q_{2}(x)=q_{1}(x) \exp \left[1-\left(1+\frac{\beta}{x}\right)^{\alpha}\right]$ for $x>0$. The random variable $X$ has pdf (3) if and only if the function $\xi$ defined in Theorem 1 has the form

$$
\xi(x)=\frac{1}{2}\left\{1+\exp \left[1-\left(1+\frac{\beta}{x}\right)^{\alpha}\right]\right\}, \quad x>0 .
$$

Proof. If $X$ has pdf (3), then

$$
(1-F(x)) E\left[q_{1}(X) \mid X \geq x\right]=1-\exp \left[1-\left(1+\frac{\beta}{x}\right)^{\alpha}\right], \quad x>0,
$$

and

$$
(1-F(x)) E\left[q_{2}(X) \mid X \geq x\right]=\frac{1}{2}\left\{1-\exp \left\{2\left[1-\left(1+\frac{\beta}{x}\right)^{\alpha}\right]\right\}\right\}, \quad x>0 .
$$

Finally

$$
\xi(x) q_{1}(x)-q_{2}(x)=\frac{1}{2} q_{1}(x)\left\{1-\exp \left[1-\left(1+\frac{\beta}{x}\right)^{\alpha}\right]\right\}
$$

for $x>0$ and hence the result.

Conversely, if $\xi$ is given as above, then

$$
s^{\prime}(x)=\frac{\xi^{\prime}(x) h(x)}{\xi(x) h(x)-g(x)}=\frac{\alpha \beta x^{-2}\left(1+\frac{\beta}{x}\right)^{\alpha-1} \exp \left[1-\left(1+\frac{\beta}{x}\right)^{\alpha}\right]}{1-\exp \left[1-\left(1+\frac{\beta}{x}\right)^{\alpha}\right]}, \quad x>0,
$$

and hence

$$
s(x)=-\log \left\{\left\{1-\exp \left[1-\left(1+\frac{\beta}{x}\right)^{\alpha}\right]\right\}\right\}, \quad x>0 .
$$

Now, in view of Theorem 1, $X$ has density (3).

Corollary 1: Let $X: \Omega \rightarrow(0, \infty)$ be a continuous random variable and let $q_{1}(x)$ be as in Proposition 1 . Then, $X$ has pdf (3) if and only if there exist functions $q_{2}$ and $\xi$ defined in Theorem 1 satisfying the differential equation

$$
\frac{\xi^{\prime}(x) h(x)}{\xi(x) h(x)-g(x)}=\frac{\alpha \beta x^{-2}\left(1+\frac{\beta}{x}\right)^{\alpha-1} \exp \left[1-\left(1+\frac{\beta}{x}\right)^{\alpha}\right]}{1-\exp \left[1-\left(1+\frac{\beta}{x}\right)^{\alpha}\right]}, \quad x>0 .
$$

Corollary 2: The general solution of the differential equation in Corollary 1 is

$$
\xi(x)=\left\{1-\exp \left[1-\left(1+\frac{\beta}{x}\right)^{\alpha}\right]\right\}^{-1}\left[\begin{array}{c}
-\int \alpha \beta x^{-2}\left(1+\frac{\beta}{x}\right)^{\alpha-1} \times \\
\exp \left[1-\left(1+\frac{\beta}{x}\right)^{\alpha}\right]\left(q_{1}(x)\right)^{-1} q_{2}(x)+D
\end{array}\right],
$$

where $D$ is a constant. Note that a set of functions satisfying the above differential equation is given in Proposition 1 with $D=\frac{1}{2}$. However, it should be also noted that there are other triplets $\left(q_{1}, q_{2}, \xi\right)$ satisfying the conditions of Theorem 1. 


\subsection{Characterization in terms of the reversed hazard rate function}

Proposition 2: Let $X: \Omega \rightarrow(0, \infty)$ be a continuous random variable. The pdf of $X$ is (3) if and only if its reversed hazard rate function $r_{F}(x)$ satisfies the following differential equation

$$
r_{F}^{\prime}(x)+\frac{2}{x} r_{F}(x)=\alpha \beta x^{-2} \frac{\mathrm{d}}{\mathrm{d} x}\left\{\frac{\left(1+\frac{\beta}{x}\right)^{\alpha-1}\left\{1+\lambda-2 \lambda \exp \left[1-\left(1+\frac{\beta}{x}\right)^{\alpha}\right]\right\}}{1+\lambda-\lambda \exp \left[1-\left(1+\frac{\beta}{x}\right)^{\alpha}\right]}\right\}, \quad x>0
$$

with the boundary condition $\lim _{x \rightarrow \infty} r_{F}(x)=0$.

Proof. If $X$ has pdf (3), then clearly the above differential equation holds.

Now, if this differential equation holds, then

$$
\frac{\mathrm{d}}{\mathrm{d} x}\left\{x^{2} r_{F}(x)\right\}=\alpha \beta \frac{\mathrm{d}}{\mathrm{d} x}\left\{\frac{\left(1+\frac{\beta}{x}\right)^{\alpha-1}\left\{1+\lambda-2 \lambda \exp \left[1-\left(1+\frac{\beta}{x}\right)^{\alpha}\right]\right\}}{1+\lambda-\lambda \exp \left[1-\left(1+\frac{\beta}{x}\right)^{\alpha}\right]}\right\}
$$

or

$$
r_{F}(x)=\frac{\alpha \beta x^{-2}\left(1+\frac{\beta}{x}\right)^{\alpha-1}\left\{1+\lambda-2 \lambda \exp \left[1-\left(1+\frac{\beta}{x}\right)^{\alpha}\right]\right\}}{1+\lambda-\lambda \exp \left[1-\left(1+\frac{\beta}{x}\right)^{\alpha}\right]}
$$

which is the reversed hazard rate function of the TINH distribution.

Remark 1: For the special case of $\lambda=0$, namely the INH distribution, the formulas in the above subsections will be quite simplified and the differential equation in Proposition 2 will have the following simple form:

$$
r_{F}^{\prime}(x)+\frac{2}{x} r_{F}(x)=-\alpha(\alpha-1) \beta^{2} x^{-4}\left(1+\frac{\beta}{x}\right)^{\alpha-2}, \quad x>0 .
$$

\section{Maximum Likelihood Estimation}

One of the common methods of estimation is the maximum likelihood (ML) estimation. The maximum likelihood estimators (MLEs) have the advantage of being asymptotically distributed as a $k$-variate normal distribution under some regularity conditions, where $k$ is the number of parameters, see Lehmann and Casella (1998). Suppose that $\boldsymbol{\theta}=(\alpha, \beta, \lambda)$ is the vector of the unknown parameters of the TINH distribution which are to be estimated based on a random sample of size $n, X_{1}, X_{2}, \cdots, X_{n}$. Let $\boldsymbol{x}=\left(x_{1}, x_{2}, \cdots, x_{n}\right)$ denote the observed values of the random sample $\boldsymbol{X}=\left(X_{1}, X_{2}, \cdots, X_{n}\right)$. Then the log-likelihood function of $\boldsymbol{\theta}$ for the given $\boldsymbol{x}$ is

$$
\begin{aligned}
\ell(\boldsymbol{\theta} ; \boldsymbol{x}) \equiv \ell(\boldsymbol{\theta})= & n \log \alpha+n \log \beta-2 \sum_{i=1}^{n} \log x_{i}+(\alpha-1) \sum_{i=1}^{n} \log \left(1+\frac{\beta}{x_{i}}\right)+n-\sum_{i=1}^{n}\left(1+\frac{\beta}{x_{i}}\right)^{\alpha} \\
& +\sum_{i=1}^{n} \log \left(1+\lambda-2 \lambda t_{i}\right)
\end{aligned}
$$

where

$$
t_{i}=\exp \left[1-\left(1+\frac{\beta}{x_{i}}\right)^{\alpha}\right]
$$

The ML estimates of the parameters are obtained by maximizing the log-likelihood function with respect to the parameters. To this end, we take the partial derivatives of $\ell(\boldsymbol{\theta})$ with respect to the unknown parameters and then set them equal to zero. Therefore, the solutions of the following nonlinear equations can help us find the required ML 
estimates.

$$
\begin{aligned}
\frac{\partial \ell(\boldsymbol{\theta})}{\partial \alpha} & =\frac{n}{\alpha}+\sum_{i=1}^{n} \log \left(1+\frac{\beta}{x_{i}}\right)-\sum_{i=1}^{n}\left(1+\frac{\beta}{x_{i}}\right)^{\alpha} \log \left(1+\frac{\beta}{x_{i}}\right)-2 \lambda \sum_{i=1}^{n} \frac{t_{i}^{(\alpha)}}{1+\lambda-2 \lambda t_{i}}=0, \\
\frac{\partial \ell(\boldsymbol{\theta})}{\partial \beta} & =\frac{n}{\beta}+(\alpha-1) \sum_{i=1}^{n} \frac{1}{x_{i}+\beta}-\alpha \sum_{i=1}^{n} \frac{1}{x_{i}}\left(1+\frac{\beta}{x_{i}}\right)^{\alpha-1}-2 \lambda \sum_{i=1}^{n} \frac{t_{i}^{(\beta)}}{1+\lambda-2 \lambda t_{i}}=0, \\
\frac{\partial \ell(\boldsymbol{\theta})}{\partial \lambda} & =\sum_{i=1}^{n} \frac{1-2 t_{i}}{1+\lambda-2 \lambda t_{i}}=0,
\end{aligned}
$$

where

$$
t_{i}^{(\alpha)}=\frac{\partial t_{i}}{\partial \alpha}=-\left(1+\frac{\beta}{x_{i}}\right)^{\alpha} \exp \left[1-\left(1+\frac{\beta}{x_{i}}\right)^{\alpha}\right] \log \left(1+\frac{\beta}{x_{i}}\right)
$$

and

$$
t_{i}^{(\beta)}=\frac{\partial t_{i}}{\partial \beta}=-\frac{\alpha}{x_{i}}\left(1+\frac{\beta}{x_{i}}\right)^{\alpha-1} \exp \left[1-\left(1+\frac{\beta}{x_{i}}\right)^{\alpha}\right] .
$$

Computational softwares may help us to find the solutions of the above equations. Next, we consider the asymptotic behavior of the MLEs for the purpose of the interval estimation. As stated earlier, MLEs are asymptotically normally distributed under some regularity conditions. Let $\widehat{\alpha}, \widehat{\beta}$ and $\widehat{\lambda}$ denote the MLEs of $\alpha, \beta$ and $\lambda$, respectively, then as $n \longrightarrow \infty$, we have

$$
(\widehat{\alpha}-\alpha, \widehat{\beta}-\beta, \widehat{\lambda}-\lambda)^{T} \stackrel{D}{\longrightarrow} N_{3}\left(\mathbf{0}, \mathbf{J}_{n}^{-1}(\boldsymbol{\theta})\right),
$$

where $\stackrel{D}{\longrightarrow}$ means convergence in distribution, $\mathbf{0}$ is a vector whose elements are all equal to zero, $\mathbf{J}_{n}(\boldsymbol{\theta})$ is the expected Fisher information matrix contained in the random sample $X_{1}, X_{2}, \cdots, X_{n}$ and $\mathbf{J}_{n}^{-1}(\boldsymbol{\theta})$ is the inverse matrix of $\mathbf{J}_{n}(\boldsymbol{\theta})$. We may use an estimator of $\mathbf{J}_{n}^{-1}(\boldsymbol{\theta})$ to find the asymptotic confidence intervals. Consider the following matrix

$$
\mathbf{I}_{n}(\boldsymbol{\theta})=-\left[\begin{array}{ccc}
I_{\alpha \alpha} & I_{\alpha \beta} & I_{\alpha \lambda} \\
I_{\beta \alpha} & I_{\beta \beta} & I_{\beta \lambda} \\
I_{\lambda \alpha} & I_{\lambda \beta} & I_{\lambda \lambda}
\end{array}\right]=-\left[\begin{array}{ccc}
\frac{\partial^{2} \ell(\boldsymbol{\theta} ; \boldsymbol{X})}{\partial \alpha^{2}} & \frac{\partial^{2} \ell(\boldsymbol{\theta} ; \boldsymbol{X})}{\partial \alpha \partial \beta} & \frac{\partial^{2} \ell(\boldsymbol{\theta} ; \boldsymbol{X})}{\partial \alpha \partial \lambda} \\
\frac{\partial^{2} \ell(\boldsymbol{\theta} ; \boldsymbol{X})}{\partial \beta \partial \alpha} & \frac{\partial^{2} \ell(\boldsymbol{\theta} ; \boldsymbol{X})}{\partial \beta^{2}} & \frac{\partial^{2} \ell(\boldsymbol{\theta} ; \boldsymbol{X})}{\partial \beta \partial \lambda} \\
\frac{\partial^{2} \ell(\boldsymbol{\theta} ; \boldsymbol{X})}{\partial \lambda \partial \alpha} & \frac{\partial^{2} \ell(\boldsymbol{\theta} ; \boldsymbol{X})}{\partial \lambda \partial \beta} & \frac{\partial^{2} \ell(\boldsymbol{\theta} ; \boldsymbol{X})}{\partial \lambda^{2}}
\end{array}\right] .
$$

The observed elements of $-\mathbf{I}_{n}(\boldsymbol{\theta})$ are

$$
\begin{aligned}
& I_{\alpha \alpha}=\frac{\partial^{2} \ell(\boldsymbol{\theta})}{\partial \alpha^{2}}=\frac{-n}{\alpha^{2}}-\sum_{i=1}^{n}\left(1+\frac{\beta}{x_{i}}\right)^{\alpha}\left[\log \left(1+\frac{\beta}{x_{i}}\right)\right]^{2}-2 \lambda \sum_{i=1}^{n} \frac{t_{i}^{(\alpha \alpha)}}{1+\lambda-2 \lambda t_{i}}-4 \lambda^{2} \sum_{i=1}^{n} \frac{\left[t_{i}^{(\alpha)}\right]^{2}}{\left(1+\lambda-2 \lambda t_{i}\right)^{2}}, \\
& I_{\alpha \beta}=I_{\beta \alpha}=\frac{\partial^{2} \ell(\boldsymbol{\theta})}{\partial \alpha \partial \beta}=\sum_{i=1}^{n} \frac{1}{x_{i}+\beta}-\sum_{i=1}^{n} \frac{1}{x_{i}}\left(1+\frac{\beta}{x_{i}}\right)^{\alpha-1}\left[1+\alpha \log \left(1+\frac{\beta}{x_{i}}\right)\right]-2 \lambda \sum_{i=1}^{n} \frac{t_{i}^{(\alpha \beta)}}{1+\lambda-2 \lambda t_{i}} \\
& \quad-4 \lambda^{2} \sum_{i=1}^{n} \frac{t_{i}^{(\alpha)} t_{i}^{(\beta)}}{\left(1+\lambda-2 \lambda t_{i}\right)^{2}}, \\
& I_{\alpha \lambda}=I_{\lambda \alpha}=\frac{\partial^{2} \ell(\boldsymbol{\theta})}{\partial \alpha \partial \lambda}=-2 \sum_{i=1}^{n} \frac{t_{i}^{(\alpha)}}{\left(1+\lambda-2 \lambda t_{i}\right)^{2}}, \\
& I_{\beta \beta}=\frac{\partial^{2} \ell(\boldsymbol{\theta})}{\partial \beta^{2}}=\frac{-n}{\beta^{2}}-(\alpha-1) \sum_{i=1}^{n} \frac{1}{\left(x_{i}+\beta\right)^{2}}-\alpha(\alpha-1) \sum_{i=1}^{n} \frac{1}{x_{i}^{2}}\left(1+\frac{\beta}{x_{i}}\right)^{\alpha-2}-2 \lambda \sum_{i=1}^{n} \frac{t_{i}^{(\beta \beta)}}{1+\lambda-2 \lambda t_{i}} \\
& \quad-4 \lambda^{2} \sum_{i=1}^{n} \frac{\left[t_{i}^{(\beta)}\right]^{2}}{\left(1+\lambda-2 \lambda t_{i}\right)^{2}},
\end{aligned}
$$




$$
I_{\beta \lambda}=I_{\lambda \beta}=\frac{\partial^{2} \ell(\boldsymbol{\theta})}{\partial \beta \partial \lambda}=-2 \sum_{i=1}^{n} \frac{t_{i}^{(\beta)}}{\left(1+\lambda-2 \lambda t_{i}\right)^{2}}, \quad I_{\lambda \lambda}=\frac{\partial^{2} \ell(\boldsymbol{\theta})}{\partial \alpha^{2}}=-\sum_{i=1}^{n}\left(\frac{1-2 t_{i}}{1+\lambda-2 \lambda t_{i}}\right)^{2}
$$

where

$$
\begin{aligned}
& t_{i}^{(\alpha \alpha)}=\frac{\partial^{2} t_{i}}{\partial \alpha^{2}}=\left(1+\frac{\beta}{x_{i}}\right)^{\alpha}\left\{\left(1+\frac{\beta}{x_{i}}\right)^{\alpha}-1\right\}\left[\log \left(1+\frac{\beta}{x_{i}}\right)\right]^{2} \exp \left[1-\left(1+\frac{\beta}{x_{i}}\right)^{\alpha}\right] \\
& t_{i}^{(\alpha \beta)}=\frac{\partial^{2} t_{i}}{\partial \alpha \partial \beta}=-\frac{1}{x_{i}}\left(1+\frac{\beta}{x_{i}}\right)^{\alpha-1}\left\{1+\alpha \log \left(1+\frac{\beta}{x_{i}}\right)\left[1-\left(1+\frac{\beta}{x_{i}}\right)^{\alpha}\right]\right\} \exp \left[1-\left(1+\frac{\beta}{x_{i}}\right)^{\alpha}\right],
\end{aligned}
$$

and

$$
t_{i}^{(\beta \beta)}=\frac{\partial^{2} t_{i}}{\partial \beta^{2}}=\frac{\alpha}{x_{i}^{2}}\left(1+\frac{\beta}{x_{i}}\right)^{\alpha-2}\left\{\alpha\left(1+\frac{\beta}{x_{i}}\right)^{\alpha}-(\alpha-1)\right\} \exp \left[1-\left(1+\frac{\beta}{x_{i}}\right)^{\alpha}\right] .
$$

Let $\mathbf{I}_{n}^{-1}(\boldsymbol{\theta})$ be the inverse matrix of $\mathbf{I}_{n}(\boldsymbol{\theta})$. One may replace the parameters that appear in the elements of $\mathbf{I}_{n}^{-1}(\boldsymbol{\theta})$ with their corresponding MLEs, to obtain an estimator of $\mathbf{J}_{n}^{-1}(\boldsymbol{\theta})$, denoted by $\widehat{\mathbf{J}}_{n}^{-1}(\boldsymbol{\theta})$. Now, let $z_{\delta}$ denote the $\delta$-th upper quantile of the standard normal distribution, then the $100(1-\gamma) \%$ two-sided equi-tailed approximate confidence intervals for $\alpha, \beta$ and $\lambda$ are given by

$$
\widehat{\alpha} \pm z_{\frac{\gamma}{2}} \sqrt{\widehat{\operatorname{Var}}(\widehat{\alpha})}, \quad \widehat{\beta} \pm z_{\frac{\gamma}{2}} \sqrt{\widehat{\operatorname{Var}}(\widehat{\beta})} \quad \text { and } \quad \widehat{\lambda} \pm z_{\frac{\gamma}{2}} \sqrt{\widehat{\operatorname{Var}}(\widehat{\lambda})}
$$

respectively, where $\widehat{\operatorname{Var}}(\cdot)$ is the diagonal element of $\widehat{\mathbf{J}}_{n}^{-1}(\boldsymbol{\theta})$ corresponding to each parameter.

\subsection{A simulation study}

Here, we present a small simulation study in order to evaluate the method of ML estimation of the unknown parameters of the TINH distribution. To this end, we generate $N=1000$ random samples of sizes $n=100,200$ and 500 from the TINH distribution for selected parameter values. Then, we obtain the ML estimates in each replication. The evaluation is performed based on two criteria: the empirical bias (bias for short) and the estimated root mean squared error (ERMSE). The computations are done using R (R Core Team, 2018). We excluded a number of replications, for which the convergence was not met and/or the ML estimate of $\lambda$ did not belong to $[-1,1]$. Moreover, we encountered a few number of errors so the related replications were omitted. The numerical results are given in Table 1. From Table 1, we observe that the ML method performs rather well in the most cases.

\section{Application}

In this section, we intend to provide an application of the new model to a real data set. To this end, we consider the time interval failure data (in thousands of hours) for three secondary pumps, reported by Suprawhardana and Sangadji (1999). The data are as follows:

$$
\begin{aligned}
& 2.160,0.746,0.402,0.954,0.491,6.560,4.992,3.474,0.150,0.358,0.101,1.359,3.465 \\
& 1.060,0.614,1.921,4.082,0.199,0.605,0.273,0.070,0.062,5.320 .
\end{aligned}
$$

We compare the fit of the TINH distribution with those of the other models, listed below

- The INH distribution, introduced by Tahir et al. (2018), which is a special case of the TINH distribution with $\lambda=0$.

- The TIE distribution, discussed by Oguntunde and Adejumo (2015), which is a special case of the TINH distribution with $\alpha=1$.

- The IE distribution, which is a special case of the TINH distribution with $\lambda=0$ and $\alpha=1$.

- The transmuted inverse Weibull (TIW) distribution, discussed also by Mahmoud and Mandouh (2013), with the 
Table 1: The biases and ERMSEs of the MLEs $(\beta=1)$.

\begin{tabular}{|c|c|c|c|c|c|c|c|}
\hline \multirow[b]{3}{*}{$\alpha$} & \multirow[b]{3}{*}{$\lambda$} & \multicolumn{6}{|l|}{$n=100$} \\
\hline & & \multicolumn{3}{|l|}{ Bias } & \multicolumn{3}{|l|}{ ERMSE } \\
\hline & & $\widehat{\alpha}$ & $\widehat{\beta}$ & $\widehat{\lambda}$ & $\widehat{\alpha}$ & $\widehat{\beta}$ & $\hat{\lambda}$ \\
\hline \multirow[t]{2}{*}{0.5} & -0.5 & -0.0521 & 1.8014 & 0.7554 & 0.0944 & 2.3452 & 0.8436 \\
\hline & 0.5 & 0.0324 & 0.3044 & -0.0141 & 0.1617 & 0.9170 & 0.3870 \\
\hline \multirow[t]{2}{*}{1} & -0.5 & 0.3077 & 1.0045 & 0.4398 & 1.2656 & 1.8406 & 0.6105 \\
\hline & 0.5 & 0.2485 & 0.4137 & 0.0283 & 1.0708 & 1.0873 & 0.3535 \\
\hline \multirow[t]{4}{*}{3} & -0.5 & 9.3051 & -0.7734 & -0.1177 & 9.4159 & 0.8152 & 0.2452 \\
\hline & 0.5 & 10.7955 & -0.8259 & -0.2537 & 10.8059 & 0.8261 & 0.3779 \\
\hline & & \multicolumn{6}{|l|}{$\bar{n} n=200$} \\
\hline & & \multicolumn{3}{|l|}{ Bias } & \multicolumn{3}{|l|}{ ERMSE } \\
\hline$\alpha$ & $\lambda$ & $\widehat{\alpha}$ & $\widehat{\beta}$ & $\widehat{\lambda}$ & $\widehat{\alpha}$ & $\widehat{\beta}$ & $\widehat{\lambda}$ \\
\hline \multirow[t]{2}{*}{0.5} & -0.5 & -0.0654 & 2.0660 & 0.8272 & 0.0866 & 2.5643 & 0.8959 \\
\hline & 0.5 & 0.0088 & 0.3174 & -0.0051 & 0.1156 & 0.8828 & 0.3678 \\
\hline \multirow[t]{2}{*}{1} & -0.5 & -0.0440 & 1.3362 & 0.5482 & 0.7680 & 1.9966 & 0.6628 \\
\hline & 0.5 & 0.0549 & 0.4487 & 0.0543 & 0.5198 & 1.0168 & 0.3104 \\
\hline \multirow[t]{4}{*}{3} & -0.5 & 9.4833 & -0.8036 & -0.1882 & 9.5271 & 0.8048 & 0.2376 \\
\hline & 0.5 & 10.8783 & -0.8279 & -0.2742 & 10.8871 & 0.8281 & 0.3385 \\
\hline & & \multicolumn{6}{|l|}{$n=500$} \\
\hline & & \multicolumn{3}{|l|}{ Bias } & \multicolumn{3}{|l|}{ ERMSE } \\
\hline$\alpha$ & $\lambda$ & $\widehat{\alpha}$ & $\widehat{\beta}$ & $\widehat{\lambda}$ & $\widehat{\alpha}$ & $\widehat{\beta}$ & $\widehat{\lambda}$ \\
\hline \multirow[t]{2}{*}{0.5} & -0.5 & -0.0732 & 2.1405 & 0.8679 & 0.0830 & 2.5033 & 0.9052 \\
\hline & 0.5 & 0.0105 & 0.0934 & -0.0316 & 0.0766 & 0.5389 & 0.2749 \\
\hline \multirow[t]{2}{*}{1} & -0.5 & -0.2050 & 1.3522 & 0.5893 & 0.2917 & 1.7064 & 0.6458 \\
\hline & 0.5 & -0.0541 & 0.5527 & 0.1052 & 0.3453 & 1.0433 & 0.2641 \\
\hline \multirow[t]{2}{*}{3} & -0.5 & 9.6939 & -0.8145 & -0.2419 & 9.7098 & 0.8152 & 0.2573 \\
\hline & 0.5 & 10.9633 & -0.8293 & -0.2566 & 10.9635 & 0.8294 & 0.2850 \\
\hline
\end{tabular}

following pdf

$$
f_{T I W}(x)=\frac{\alpha \beta}{x^{\alpha+1}} \exp \left(-\frac{\beta}{x^{\alpha}}\right)\left\{1+\lambda-2 \lambda \exp \left(-\frac{\beta}{x^{\alpha}}\right)\right\}, \quad \alpha>0, \quad \beta>0, \quad|\lambda| \leq 1, \quad x>0 .
$$

- The inverse Weibull (IW) distributionwhich is a special case of the TIW distribution with $\lambda=0$.

We obtain the ML estimates of the parameters of the above models. We shall use some goodness-of-fit criteria such as the minimum value of the minus $\log$-likelihood $(-\log (L))$, Anderson-Darling statistic $(A *)$, Cramér-von Mises statistic $\left(W^{*}\right)$, Kolmogorov-Smirnov (K-S) test statistic and its corresponding $p$-value in order to compare the fitted models. Generally, a model with smaller values of K-S test statistic, $-\log (L), A^{*}$ and $W^{*}$ possesses a better fit. For details pertaining to $A^{*}$ and $W^{*}$, one may refer to Chen and Balakrishnan (1995). The numerical computations were performed using R (R Core Team, 2018) and the package AdequacyModel, see Marinho et al. (2013). The results are summarized in Table 2. For visual comparison, the empirical histogram of the data, as well as the fitted pdfs of the considered models, are displayed in Figure 7. Moreover, the probability-probability (P-P) plots of the considered models are illustrated in Figure 8.

From Table 2, it can be observed that the smallest values of K-S test statistic, $-\log (L), A^{*}$ and $W^{*}$ belong to the TINH distribution, therefore the TINH distribution provides the best fit among all the considered models. From Figures 7 and 8 , we can conclude that the TINH distribution seems quite suitable for the time interval failure data, as well. To sum up, the fit of the TINH model is superior to those of the considered distributions. 
Table 2: Parameter ML estimates (standard errors in the parentheses) and the goodness-of-fit test statistics.

\begin{tabular}{lllllllll}
\hline Model & $\alpha$ & $\beta$ & $\lambda$ & $-\log (L)$ & $W^{*}$ & $A^{*}$ & K-S & $p$-value \\
\hline TINH & 0.4689 & 1.2843 & -0.2025 & 35.2647 & 0.0380 & 0.3291 & 0.0993 & 0.9604 \\
& $(0.1565)$ & $(1.6183)$ & $(0.9637)$ & & & & & \\
INH & 0.4510 & 1.6402 & & 35.2812 & 0.0387 & 0.3334 & 0.1005 & 0.9563 \\
& $(0.1260)$ & $(1.1122)$ & & & & & & \\
TIE & & 0.2498 & -0.5420 & 36.9721 & 0.0702 & 0.5282 & 0.1679 & 0.4845 \\
& & $(0.0725)$ & $(0.3279)$ & & & & & \\
IE & & & & 38.1047 & 0.0869 & 0.6279 & 0.2014 & 0.2703 \\
& & 0.3165 & & & & & & \\
TIW & 0.8035 & 0.3531 & -0.4603 & 35.8823 & 0.0580 & 0.4550 & 0.10685 & 0.9305 \\
& $(0.1269)$ & $(0.1636)$ & $(0.5431)$ & & & & & \\
IW & 0.7489 & 0.4897 & & 36.1377 & 0.0661 & 0.5044 & 0.1128 & 0.8999 \\
& $(0.1175)$ & $(0.1368)$ & & & & & &
\end{tabular}
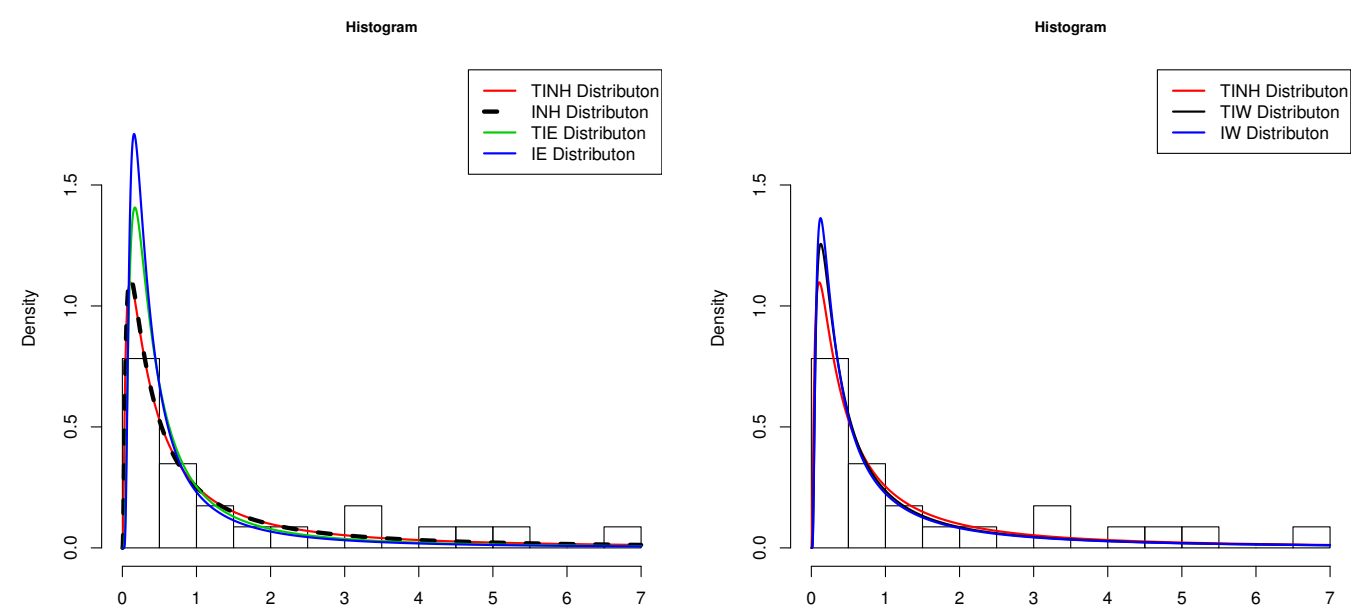

Figure 7: Empirical histogram of the data as well as the fitted pdfs of the TINH, INH, TIE, IE, TIW and IW distributions.

\section{Concluding Remarks}

In this paper, we have introduced a new three-parameter distribution called the transmuted inverted Nadarajah-Haghighi distribution. The new distribution includes the inverted Nadarajah-Haghighi distribution, the transmuted inverted exponential distribution and the inverted exponential distribution as its special cases. Several mathematical properties of the proposed distribution such as the quantile function, skewness, kurtosis, moments, reversed hazard rate function, the reliability parameter and order statistics, have been discussed. Certain characterizations of the new distribution are presented. The maximum likelihood procedure has been utilized to estimate the parameters and a small simulation study is provided to assess the procedure. The usefulness of the new distribution has been demonstrated via a real data application. All the computations are performed using Maple 17 and R (R Core Team, 2018).

From the results of the paper, we may conclude that the new distribution provides more flexibility than the other considered models. Therefore, we hope that the proposed model can be applied to lifetime (and other types of) data analysis plans. 

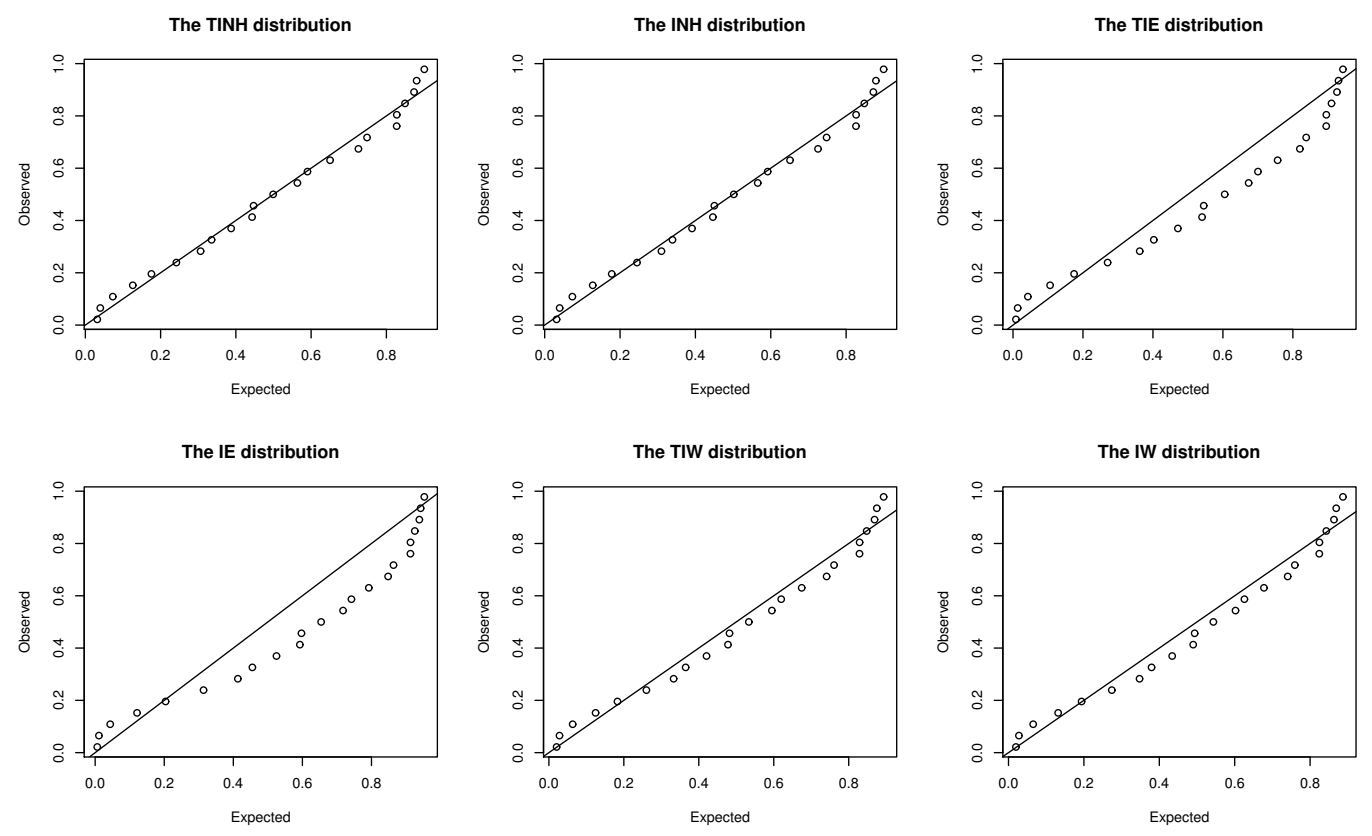

Figure 8: The P-P plots of the considered models.

\section{References}

1. Abdul-Moniem, I. B. (2015). Transmuted Burr type III distribution. Journal of Statistics: Advances in Theory and Applications, 14:37-47.

2. Block, H. W., Savits, T. H., and Singh, H. (1998). The reversed hazard rate function. Probability in the Engineering and Informational Sciences, 12:69-90.

3. Bourguignon, M., Leão, J., Leiva, V., and Santos-Neto, M. (2017). The transmuted Birnbaum-Saunders distribution. REVSTAT-Statistical Journal, 15:601-628.

4. Chen, G. and Balakrishnan, N. (1995). A general purpose approximate goodness-of-fit test. Journal of Quality Technology, 27:154-161.

5. Dimitrakopoulou, T., Adamidis, K., and Loukas, S. (2007). A lifetime distribution with an upside-down bathtub-shaped hazard function. IEEE Transactions on Reliability, 56:308-311.

6. Elbatal, I. and Aryal, G. (2015). Transmuted Dagum distribution with applications. Chilean Journal of Statistics, 6:31-45.

7. Glänzel, W. (1987). A characterization theorem based on truncated moments and its application to some distribution families, In: P. Bauer, F. Konecny, W. Wertz (Eds.), Mathematical Statistics and Probability Theory Vol. B, Springer, D. Reidel Publishing Company Dordrecht-Holland, pp. 75-84, https://doi.org/10.1007/97894-009-3965-3_8.

8. Glänzel, W. (1990). Some consequences of a characterization theorem based on truncated moments. Statistics, 21:613-618.

9. Khan, M. S. and King, R. (2014). Transmuted generalized inverse Weibull distribution. Journal of Applied Statistical Science, 20:15-32.

10. Lee, C., Famoye, F., and Alzaatreh, A. Y. (2013). Methods for generating families of univariate continuous distributions in the recent decades. WIREs Computational Statistics, 5:219-238.

11. Lehmann, E. L. and Casella, G. (1998). Theory of Point Estimation. Second ed., Springer, New York, USA.

12. Mahmoud, M. R. and Mandouh, R. M. (2013). On the transmuted Fréchet distribution. Journal of Applied Sciences Reserach, 9:5553-5561.

13. Marinho, P. R. D., Bourguignon, M., and Dias, C. R. B. (2013). AdequacyModel: Adequacy of probabilistic models and generation of pseudo-random numbers. R package version 1.0.8., https://CRAN.Rproject.org/package=AdequacyModel.

14. Merovci, F. (2013a). Transmuted exponentiated exponential distribution. Mathematical Sciences And Applications E-Notes, 1:112-122.

15. Merovci, F. (2013b). Transmuted Lindley Distribution. International Journal of Open Problems in Computer 
Science and Mathematics, 6:63-72.

16. Merovci, F., Elbatal, I., and Ahmed, A. (2013). Transmuted generalized inverse Weibull distribution. arXiv preprint arXiv:1309.3268.

17. Moors, J. J. A. (1988). A quantile alternative for kurtosis. Journal of the Royal Statistical Society: Series D (The Statistician), 37:25-32.

18. Nadarajah, S. and Haghighi, F. (2011). An extension of the exponential distribution. Statistics, 45:543-558.

19. Oguntunde, P. E. and Adejumo, A. O. (2015). The transmuted inverse exponential distribution. International Journal of Advanced Statistics and Probability, 3:1-7.

20. R Core Team (2018). R: A Language and Environment for Statistical Computing. R Foundation for Statistical Computing, Vienna, Austria.

21. Shaw, W. T. and Buckley, I. R. C. (2007). The alchemy of probability distributions: Beyond Gram-Charlier $\&$ Cornish-Fisher expansions, and skew-normal or kurtotic-normal distributions. Submitted.

22. Shaw, W. T. and Buckley, I. R. C. (2009). The alchemy of probability distributions: beyond GramCharlier expansions, and a skew-kurtotic-normal distribution from a rank transmutation map. arXiv preprint arXiv:0901.0434.

23. Suprawhardana, M. S. and Sangadji, P. (1999). Total time on test plot analysis for mechanical components of the RSG-GAS reactor. Atom Indones, 25:81-90.

24. Tahir, M. H., Cordeiro, G. M., Ali, S., Dey, S., and Manzoor, A. (2018). The inverted Nadarajah-Haghighi distribution: estimation methods and application. Journal of Statistical and Computation and Simulation, 88:2775-2798. 